\title{
Correspondence
}

\section{An Empirical Measure of Element Contribution in Neural Networks}

\author{
Brenda Mak and Robert W. Blanning
}

\begin{abstract}
A frequent complaint about neural net models is that they fail to explain their results in any useful way. The problem is not a lack of information, but an abundance of information that is difficult to interpret. When trained, neural nets will provide a predicted output for a posited input, and they can provide additional information in the form of interelement connection strengths. But this latter information is of little use to analysts and managers who wish to interpret the results they have been given. In this paper, we develop a measure of the relative importance of the various input elements and hidden layer elements, and we use this to interpret the contribution of these components to the outputs of the neural net.
\end{abstract}

Index Terms-Clustering methods, hidden element contribution, input element contribution, measurement index, neural network architecture.

\section{INTRODUCTION}

During the past few years, a growing number of researchers and practitioners have been applying neural networks to analysis and decision support for problems in business, engineering, medicine, communications, and other areas [22], [34], [36]. A limitation of these systems is that they provide no explanation as to why a particular conclusion is reached [13], [35], [39]. In other words, neural nets may give accurate predictions of future events or recommend decisions that turn out to be reasonable, but they cannot explain how they arrived at the results or why they should be trusted.

Many users find this disconcerting, especially since other decision support tools have at least some explanatory capability. Causal decision models, such as mathematical programming models and simulations, can provide information describing the consequences of a change in a proposed parameter, and certain intelligent systems can call on their internal symbolic structures to explain the chain of reasoning that led to a particular conclusion.

Several attempts have been made to provide the same functionality in neural nets. Measures of the relative importance [13] or relative strength [39] of inputs to the net have been developed. If the connection strengths associated with a particular input and output are large relative to the other weights, these measures report a high degree of importance or strength. Attempts have also been made to assign meanings to the processing elements in the hidden layers, although this is far more difficult [37].

Our purpose is to extend this type of analysis in the context of a neural net model of new product entry decisions. Section II reviews the studies on neural networks and the existing relative importance measures for neural net inputs. In Section III, we introduce a measure that approximates the rate of change of the output of the net with respect to its input, and a disaggregation of this measure to interpret the contribution of the hidden layer elements. In Section IV, we

Manuscript received February 14, 1996; revised April 20, 1997 and January 15, 1998.

B. Mak is with the Department of Computer Science and Information Systems, the University of Hong Kong, Pokfulam, Hong Kong (e-mail: bmak@csis.hku.hk).

R. W. Blanning is with the Owen Graduate School of Management, Vanderbilt University, Nashville, TN 37203 USA, and the Hong Kong University of Science and Technology, Clear Water Bay, Hong Kong.

Publisher Item Identifier S 1094-6977(98)07475-6. apply the methods discussed in Sections II and III to an important management problem - the introduction of new products.

\section{Neural Net Models and Contribution Measures}

Consider a neural network with one hidden layer of elements, with $I$ input elements, $J$ elements in the hidden layer, and $K$ output elements. The inputs $x_{i}, i=1 \cdots I$ are entered into the network. Each hidden layer element transfers a weighted sum $y_{j}=\Sigma_{i=1} w_{j i} x_{i}+\theta_{j}$ into $z_{j}$, where $z_{j}=1 /\left(1+\exp \left(-y_{j}\right)\right)$. The $w_{j i}$ are the connection strengths between the input and the hidden layer and $\theta_{j}$ is a bias. At the output layer $k=1 \cdots K$, the weighted sums $u_{k}=\Sigma_{j=1} v_{j k} z_{j}+$ $\tau_{k}$ are then transformed into $s_{k}=1 /\left(1+\exp \left(-u_{k}\right)\right)$. The $v_{j k}$ are the connection strengths between the hidden layer elements and the output layer, and $\tau_{k}$ is a bias. The final binary output $c_{k}$ is one if $s_{k}$ is above a threshold value, otherwise it is zero. The hidden layers are used to model the nonlinearities in the relationship between inputs and output [20]. For simple continuous functions, one hidden layer with a sufficient number of elements may be appropriate [10], [18], but for more complicated cases, two or more hidden layers may be required [9]. The neural net method is more robust and has better predictive accuracy than classical statistical methods, such as discriminant and logit analysis, in bond rating prediction and classification [12], [36], mortgage underwriting judgments [8], corporate failure prediction [27], and bank bankruptcy prediction [35].

Neural nets are often trained using a backpropagation algorithm [29], which is simple and easy to compute [25]. It often converges rapidly to a local minimum [16], [22], but it may not find a global minimum and in some cases may not converge at all [14], [17]. To overcome this problem, a momentum term is often added to the minimization function [7], [29] and a variable learning rate is applied [38].

Neural networks have been criticized for their lack of explanatory capability [35]. Unlike traditional statistical methods, it is difficult to interpret the significance of the input variables and understand the role played by the elements in the hidden layer. Various researchers have attempted to identify the contribution of various components of the network [15], [26], [32], [33], [36]. For example, Barlett [2] used entropy to compute the Information Theoretic Interdependency Analysis (ITIA) to measure the association between the input and output of the net. In order to assess the contribution of the input variables, Yoon et al. [39], [40] and Garson [13] have developed measures based on the $w_{j i}$ and $v_{j k}$ connection strengths when the net stabilizes in training. For the $k$ th output, $i$ th input, and $j$ th elements in the hidden layer, the Yoon et al. measure of the relative contribution of input $i$ on output $k$ is

$$
\operatorname{Con}_{i k}=\frac{\sum_{j-1}^{J} w_{j i} v_{j k}}{\sum_{i=1}^{I}\left|\sum_{j=1}^{J} w_{j i} v_{j k}\right|} .
$$

Garson's method places more emphasis on the connection strengths from the hidden layer to the output layer (i.e., the $v_{j k}$ ), but it does not measure the direction of influence (positive or negative). Garson's 
measure is

$$
\operatorname{Con}_{i k}=\frac{\sum_{j=1}^{J} \frac{\left|w_{j i}\right|\left|v_{j k}\right|}{\sum_{i=1}^{I}\left|w_{j i}\right|}}{\sum_{i=1}^{I} \sum_{j=1}^{J} \frac{\left|w_{j i}\right|\left|v_{j k}\right|}{\sum_{i=1}^{I}\left|w_{j i}\right|}} .
$$

Both of these measures incorporate certain rates of change of the strengths of signals as they flow through the network. For example, $w_{j i}=\delta y_{i} / \delta x_{i}$, that is, the partial derivatives of the inputs to the hidden layer with respect to the inputs to the network. Similarly, $v_{j k}=\delta u_{k} / \delta z_{j}$, that is, the partial derivatives of the inputs to the output layer with respect to the outputs of the hidden layer. But neither method includes $\delta z_{j} / \delta y_{j}$, that is, the partial derivatives of the output of the hidden layer elements with respect to the inputs to the hidden layer elements. In other words, neither Yoon et al. nor Garson addresses the rates of change across the hidden layer. We will show how to do this and incorporate it into a more comprehensive measure of contribution of network inputs to outputs.

\section{New Measures for CONTRIBUtion Analysis}

In this section, we develop a new measure for measuring the contribution of the inputs to the output. This new measure takes into consideration the contribution of the elements in the hidden layer. In addition, we develop measures to assess the contribution of the elements in the hidden layer and examine the clustering effect of the elements in the hidden layer.

When we consider the contribution of the input $x_{i}$ to the output $s_{k}$ through the elements in the hidden layer, the overall contribution measure as derived by the chain rule is

$$
\frac{\delta s_{k}}{\delta x_{i}}=\sum_{j=1}^{J} \frac{\delta s_{k}}{\delta u_{k}} \cdot \frac{\delta u_{k}}{\delta z_{j}} \cdot \frac{\delta z_{j}}{\delta y_{j}} \cdot \frac{\delta y_{j}}{\delta x_{i}} .
$$

Substituting for the derivatives in this expression, we obtain

$$
\frac{\delta s_{k}}{\delta x_{i}}=s_{k}\left(1-s_{k}\right) \sum_{j=1}^{J} v_{j k} z_{j}\left(1-z_{j}\right) w_{j i} .
$$

However, each $z_{j}\left(1-z_{j}\right)$, the rate of change of output of the $j$ th hidden element with respect to its input. We will call this $\beta_{j}$. To estimate $\beta_{j}$, we will average $z_{j}\left(1-z_{j}\right)$ across the trials of the training set. If $t=1 \cdots T$ denotes the trials and $z_{j t}$ is the value of the $j$ th hidden layer output during the $t$ th trial, then

$$
\beta_{j}=\frac{1}{T} \sum_{t=1}^{T} z_{j t}\left(1-z_{j t}\right) .
$$

This type of simple approximation to a more complex model is sometimes called a metamodel [1], [6] or a heuristic model [5].

In order to obtain a measure of the relative contribution of the $i$ th input to the $k$ th output, we divide the partial derivative from (4) by the sum of the absolute values of the partial derivatives for all inputs. We will call this $\mathrm{INC}_{i k}$ for the input contribution, similar to the $\mathrm{CON}_{i k}$ measures developed by Garson and Yoon et al. This results in the following measure of relative contribution of input element $i$ on output element $k$ :

$$
\mathrm{INC}_{i k}=\frac{\sum_{j=1}^{J} v_{j k} \beta_{j} w_{j i}}{\sum_{i=1}^{I}\left|\sum_{j=1}^{J} v_{j k} \beta_{j} w_{j i}\right|} .
$$

TABLE I

Decision Variables and VAlues

\begin{tabular}{l|l|l}
\hline \hline Variable & Variable Definition & Values and Meanings \\
\hline $\mathrm{x}_{1}$ & position of the firm & small (0) versus dominant (1) \\
\hline $\mathrm{x}_{2}$ & financial strength of the firm & weak (0) versus strong (1) \\
\hline $\mathrm{x}_{3}$ & expected demand growth & low $(0)$ versus high (1) \\
\hline $\mathrm{x}_{4}$ & product life cycle & short (0) versus long (1) \\
\hline $\mathrm{x}_{5}$ & diffusion rate across competitors & low (0) versus high (1) \\
\hline $\mathrm{x}_{6}$ & cannibalization & low (0) versus high (1) \\
\hline $\mathrm{x}_{7}$ & cost of market development & low (0) versus high (1) \\
\hline $\mathrm{c}$ & product entry decision & No-Go (0) versus Go (1) \\
\hline \hline
\end{tabular}

This is an enhancement of the Yoon et al. and Garson measures in two respects. First, it considers the rate of change across the hidden layer. Second, it can be disaggregated to measure the contribution of each hidden layer element $j$ to the contribution of input element $i$ to output element $k$. Thus, we disaggregate the input contribution measure $\mathrm{INC}_{i k}$ to obtain the hidden layer contribution measure for the $j$ th hidden layer element, as follows:

$$
\mathrm{HLC}_{i j k}=\frac{v_{j k} \beta_{j} w_{j i}}{\sum_{i=1}^{I} \sum_{j=1}^{J}\left|v_{j k} \beta_{j} w_{j i}\right|} .
$$

We note that the sum of the hidden layer contribution measures is the input contribution measure, that is, $\mathrm{INC}_{i k}=\Sigma_{j=1}^{J} H L C_{i j k}$.

The $\mathrm{HLC}_{i j k}$ measures may be helpful in interpreting the contribution of the hidden layer elements. If the $\mathrm{HLC}_{i j k}$ measures are very large in absolute value for certain combinations of inputs and outputs, that suggests that the hidden layer element is clustering those inputs-and this may suggest an interpretation for the hidden layer element. This will be illustrated in the example below.

\section{Application to the New Product Entry Decision}

Many business organizations facing competitive pressure have to reengineer their products for survival [30]. One of the important decisions involved in the product reengineering issue is whether to introduce a new product into the market [21]. A manager needs to balance the costs and benefits involved in deciding whether to introduce the product. A premature introduction may result in loss of investment, but an unnecessary delay may result in missed opportunities. Therefore, it would be useful to have a model for new product entry. However, many contingency factors have to be considered simultaneously and little historical data is available to guide managers in analyzing these factors to make a decision. We constructed a model using judgmental data obtained from 36 senior MBA students who had an average work experience of four years and were experienced in strategic marketing analysis. The seven variables in Table I were used as a framework to elicit knowledge from the experts. The details of the knowledge acquisition process are described in [23] and [24].

We compared Yoon et al., Garson, and our measures with two multivariate statistical techniques: logit regression [11] and discriminant analysis [19] and the ID3 pattern classification method [28]. We note that a neural net with no hidden layer reduces to a discriminant analysis [20]. In the neural net, the number of elements in the hidden layer was varied to find the model with the minimum error [29], [38], which occurred when there were three elements in the hidden layer.

Table II shows the coefficients of the models developed with the logit regression method, the discriminant analysis method, and entropy reduction with the ID3 method. For the logit model, the 
TABLE II

COEFFicIENTS OF RELATIVE IMPACT

\begin{tabular}{|c|c|c|c|c|c|c|}
\hline Variables & Logit (rank) & $\begin{array}{l}\text { Discriminant Analysis } \\
\text { unstan- } \quad \text { standard- } \\
\text { dardized ized (rank) }\end{array}$ & $\begin{array}{l}\text { ID3 } \\
\text { entropy } \\
\text { reduction (rank) }\end{array}$ & $\begin{array}{l}\text { Neura } \\
\text { Yoon } \\
\text { (rank) }\end{array}$ & $\begin{array}{l}\text { I Net Moc } \\
\text { Garson } \\
\text { (rank) }\end{array}$ & $\begin{array}{l}\text { New } \\
\text { (rank) }\end{array}$ \\
\hline$\overline{x_{1}}$ & $\begin{array}{l}1.845^{*} \\
(\mathrm{t}=2.824) \quad\left(2^{\text {nd }}\right)\end{array}$ & $\begin{array}{l}1.37 \quad 0.484^{*} \\
(\mathrm{~F}=0.33, \mathrm{p}=.56)\end{array}$ & $0.082 \quad\left(2^{\text {nd }}\right)$ & $\begin{array}{l}.356 \\
\left(1^{\text {st }}\right) \\
\end{array}$ & $\stackrel{216}{.216}$ & $\begin{array}{l}.308 \\
\left(1^{\text {st }}\right)\end{array}$ \\
\hline$\overline{x_{2}}$ & $\begin{array}{ll}0.994 & \\
(t=1.676) & \left(7^{\text {th }}\right) \\
\end{array}$ & $\begin{array}{|lrl|}0.495 & 0.249 & \\
(\mathrm{~F}=0.33, p=.56) & \left(7^{\text {th }}\right) \\
\end{array}$ & $0.002 \quad\left(7^{\text {th }}\right)$ & $\begin{array}{l}.001 \\
\left(7^{41}\right) \\
\end{array}$ & $\begin{array}{l}.193 \\
\left(2^{\text {nd }}\right)\end{array}$ & $\begin{array}{l}.051 \\
\left(6^{\text {Ll }}\right) \\
\end{array}$ \\
\hline$\overline{x_{3}}$ & $\begin{array}{ll}2.085^{*} & \\
(\mathrm{t}=3.375) & \left(1^{\mathrm{st}}\right) \\
\end{array}$ & $\begin{array}{|lc|}1.369 \quad 0.632^{*} \\
(\mathrm{~F}=19.19, \mathrm{p}=.0000)\left(1^{\mathrm{st}}\right) \\
\end{array}$ & $0.123 \quad\left(1^{\mathrm{st}}\right)$ & $\begin{array}{l}.109 \\
\left(5^{\mathrm{h}}\right) \\
\end{array}$ & $\begin{array}{l}.149 \\
\left(3^{\text {rd }}\right)\end{array}$ & $\begin{array}{l}.122 \\
\left(5^{\mathrm{h}}\right) \\
\end{array}$ \\
\hline$x_{4}$ & $\begin{array}{l}1.150^{*} \\
(\mathrm{t}=2.009) \quad\left(4^{\text {th }}\right) \\
\end{array}$ & $\begin{array}{ll}0.706 \quad 0.340^{*} \\
(\mathrm{~F}=9.703, \mathrm{p}=.0024) & \left(4^{\text {th }}\right) \\
\end{array}$ & $0.065 \quad\left(3^{\text {rd }}\right)$ & $\begin{array}{l}.180 \\
\left(3^{\text {rd }}\right) \\
\end{array}$ & $\begin{array}{l}.102 \\
\left(6^{\mathrm{th}}\right)\end{array}$ & $\begin{array}{l}.162 \\
\left(3^{\text {rd }}\right) \\
\end{array}$ \\
\hline$\overline{x_{5}}$ & $\begin{array}{l}-1.052 \\
(\mathrm{t}=-1.762)\left(6^{\mathrm{th}}\right) \\
\end{array}$ & $\begin{array}{ll}-0.529 \quad-0.258^{*} \\
(\mathrm{~F}=5.740, \mathrm{p}=.0185)\left(6^{\text {th }}\right)\end{array}$ & 0.04 & $\begin{array}{l}-.017 \\
\left(6^{\left(t^{\prime}\right.}\right) \\
\end{array}$ & $\begin{array}{l}.137 \\
\left(4^{4 h}\right) \\
\end{array}$ & $\begin{array}{l}-.041 \\
\left(7^{\text {th }}\right) \\
\end{array}$ \\
\hline$\overline{\mathbf{x}_{6}}$ & $\begin{array}{l}-1.173 \\
(\mathrm{t}=-1.927)\left(5^{\mathrm{th}}\right)\end{array}$ & $\begin{array}{ll}-0.562 & -0.280 \\
(\mathrm{~F}=5.74, \mathrm{p}=1368) & \left(5^{\text {th }}\right)\end{array}$ & $0.016 \quad\left(6^{\text {th }}\right)$ & $\begin{array}{l}-.152 \\
\left(4^{\text {th }}\right) \\
\end{array}$ & $\begin{array}{l}.105 \\
\left(5^{\text {th }}\right) \\
\end{array}$ & $\begin{array}{l}-.129 \\
\left(4^{\mathrm{hb}}\right) \\
\end{array}$ \\
\hline$\overline{\mathrm{x}_{7}}$ & $\begin{array}{l}-1.589^{*} \\
(\mathrm{t}=-2.597)\left(3^{\mathrm{rd}}\right)\end{array}$ & $\begin{array}{ll}-0.908 & -0.440^{*} \\
(\mathrm{~F}=4.877, \mathrm{p}=.0296) & \left(3^{\mathrm{rd}}\right)\end{array}$ & $0.034 \quad\left(4^{\text {th }}\right)$ & $\begin{array}{l}-.186 \\
\left(2^{\text {nd }}\right)\end{array}$ & $\begin{array}{l}.098 \\
\left(7^{\text {th }}\right) \quad(2\end{array}$ & $\begin{array}{l}-.186 \\
\left(2^{\text {nd }}\right) \\
\end{array}$ \\
\hline
\end{tabular}

*significant at the 0.05 level

(rank) indicates the rank of importance of the variable

dependent variable is the probability of choosing a Go strategy versus a No-Go strategy, and the independent variables are the seven binary input variables listed in Table I. As indicated by the size of the regression coefficients, $x_{3}$ (expected demand growth), $x_{1}$ (position of the firm), and $x_{7}$ (cost of market development) have the highest importance in affecting the choice of the Go strategy versus the No-Go strategy. In the discriminant analysis case, $x_{3}$ is the most important discriminant variable, followed by $x_{1}$ and $x_{7}$. This matches with the results from the logit analysis. For the ID3 method, $x_{3}$ has the highest entropy reduction and is the most important variable, followed by $x_{1}, x_{4}$, and $x_{7}$. Therefore, all three methods have identified $x_{3}$ as the most important variable, followed by $x_{1}$.

Table II shows the relative contribution measures for the Yoon et al. (1), Garson (2), and our new method (6). For all three neural net measures, $x_{1}$ has the highest contribution. For both our measure and in Yoon et al.'s measure, $x_{7}$ was second, whereas in Garson's method, $x_{2}$ was second. Thus, the logit, discriminant analysis and ID3 methods identify $x_{3}$ as the most important variable, whereas the three neural net methods all identify $x_{1}$ as the most important variable.

Although the reason for the differences are not clear, we should note that logit and discriminant analysis are both multivariate statistical methods, and it is not surprising that they produce similar results, whereas ID3 is a pattern classification method based on entropy reduction. It is also not surprising that our method and Yoon et al.'s method produce similar results since in both cases only the denominators are normalized, whereas Garson normalizes both numerator and denominator. On the other hand, the three neural net measures provide quite different results from the other three methods, possibly because of their nonlinearity and additional partitioning of the input space by the hidden layer elements [31], [38].

We use the disaggregated $\mathrm{HLC}_{i j k}$ measure from (7) to determine whether the hidden layer elements are identifying factors relating the input to the output. The left half of Table III shows the $\mathrm{HLC}_{i j k}$ measures for the neural net with three hidden layer elements and their sum across the three-hidden-elements, which are the measures of relative contribution derived in Section III. These may be interpreted as follows.

- Major contributors to the first hidden layer element $y_{1}$ are $x_{3}$ (positive contribution) and $x_{4}$ (positive contribution). Thus, the first hidden layer element makes its strongest contribution to the Go decision when expected demand growth is high and product lifecycle is long. These two variables collectively can be interpreted as market attractiveness.
TABLE III

Neural Net Importance LoAdings and Factor Scores

\begin{tabular}{|c|c|c|c|c|c|c|c|}
\hline \multirow[t]{2}{*}{ Variable } & \multicolumn{4}{|c|}{$\begin{array}{l}\text { Importance Loadings } \\
\text { (3 hidden elements) }\end{array}$} & \multicolumn{3}{|c|}{$\begin{array}{c}\text { Factor Score Coefficient Matrix } \\
\text { (3 factors) }\end{array}$} \\
\hline & $j=1$ & $\mathrm{j}=2$ & $\mathrm{j}=3$ & Sum & Factor 1 & Factor 2 & Factor 3 \\
\hline$x_{1}$ & 0.099 & 0.404 & -0.194 & .308 & -.119 & .582 & -.185 \\
\hline $\mathrm{x}_{2}$ & 0.050 & -0.279 & 0.280 & .051 & .179 & -.038 & .638 \\
\hline$x_{3}$ & 0.260 & -0.175 & 0.037 & .122 & .377 & .053 & .032 \\
\hline$x_{4}$ & 0.127 & 0.115 & -0.079 & .162 & .522 & -.232 & .107 \\
\hline $\mathbf{x}_{5}$ & -0.125 & 0.197 & -0.113 & -.041 & .010 & -.517 & -.199 \\
\hline$x_{6}$ & -0.072 & -0.159 & 0.102 & -.129 & .002 & -.039 & .401 \\
\hline $\mathrm{x}_{7}$ & 0.064 & -0.169 & -0.081 & -.186 & -.198 & .271 & .461 \\
\hline
\end{tabular}

- Major contributors to the second hidden layer element $y_{2}$ are $x_{1}$ (positive contribution) and $x_{2}$ (negative contribution). Thus, the second hidden layer element makes its strongest contribution to the Go decision when the firm is dominant in its industry but is financially weak.

- Major contributors to the third hidden layer element $y_{3}$ are $x_{2}$ (positive contribution) and $x_{1}$ (negative contribution). This is the reverse of the second hidden layer element; that is, the third element makes its major contribution to the Go decision when the firm is financially strong but not dominant in its industry.

- Effects of cannibalization $\left(x_{6}\right)$ and the cost of market development $\left(x_{7}\right)$ are diffused throughout the three hidden layer elements. Although their overall measure of relative contribution is high, they did not have a major contribution on any single hidden layer element.

This method provides a clustering of inputs across the hidden layer. The first hidden layer element corresponds to two input elements $\left(x_{3}\right.$ and $x_{4}$ ), which can be interpreted as market attractiveness. But the other two hidden layer elements distinguish two instances in which the companies offering the new product are either 1) dominant in their industry but financially weak or 2) financially strong but not dominant. Thus, the neural net is responding to both a distinctive grouping of inputs that can be interpreted as market attractiveness and a more subtle differentiation between two stereotypical firms-an industry leader with limited financial resources and a resource-rich firm entering a market in which it is not established. Financial resources help the latter overcome entry barriers and pass gateways to enter the market [4]. 
Finally, we compared the clustering effect of the hidden layer elements in the neural net with a factor analysis using principal component extraction with varimax rotation [3]. Hidden layer elements correspond to factors and the $\mathrm{HLC}_{i j k}$ measures correspond to factor score coefficients. The right half of Table III shows the factor score coefficients. The first factor, which may be interpreted as "market attractiveness," captures the contribution of $x_{3}$ (expected demand growth) and $x_{4}$ (product lifecycle); the second factor, which may be interpreted as "competitive considerations," captures the positive contribution of $x_{1}$ (position of the firm) and the negative contribution of $x_{5}$ (diffusion rate across competitors); and the third factor, which may be interpreted as cost issues, captures the positive contribution of $x_{2}$ (financial strength of the firm), $x_{6}$ (cannibalization), and $x_{7}$ (cost of market development). Factor analysis provides a different clustering of inputs than the neural net method. The first factor is similar to the first hidden layer element. However, instead of providing a subtle differentiation between two stereotypical firms, the other two factors capture the contributions of competitive considerations and cost issues. The difference might be due to the fact that factor analysis is not a predictive technique; it does not consider the output of the process, that is, the Go/No-Go decisions. Rather it clusters the inputs into similar groups. The $\mathrm{HLC}_{i j k}$ measures, on the other hand, are based on the relationships between inputs and outputs in a predictive model, as mediated by the hidden layer elements. In other words, the $\mathrm{HLC}_{i j k}$ measures are based not only on the inputs, but on how the inputs affect the outputs.

\section{CONCLUSION}

We have developed a method for determining the relative contribution of each input of a neural net on the output, and we have applied this method to a neural net model of new product entry. Our method differs from other neural net contribution measures in that it considers all components of the network, including the elements in the hidden layer, and it allows us to disaggregate the contribution measures to determine the contributions of the elements in the hidden layer. This may help us to interpret the contribution of each hidden layer element and thus gain some insight into the reasoning process modeled by the network.

\section{REFERENCES}

[1] S. C. Agrawal, Metamodeling: A Study of Approximations in Queuing Models. Cambridge, MA: MIT Press, 1985.

[2] E. Bartlett, "Self determination of input variable importance using neural networks," Neural, Parallel Sci. Computat., vol. 2, pp. 103-114, 1994.

[3] D. J. Bartholomew, Latent Variable Models and Factor Analysis. London, U.K.: Griffin, 1987, pp. 58-65.

[4] B. Benson, A. P. Sage, and G. Cook, "Emerging technology-evaluation methodology: With application to micro-electromechanical systems," IEEE Trans. Eng. Manag., vol. 40, pp. 114-123, Feb. 1993.

[5] R. W. Blanning and R. H. Crandall, "Heuristic modeling and technological impact analysis," Technol. Forecasting Social Change, vol. 15, pp. 259-271, 1979.

[6] R. W. Blanning, "The construction and implementation of metamodels," Simulation, vol. 24, pp. 177-184, June 1976.

[7] M. Chester, Neural Networks-A Tutorial. Englewood Cliffs, NJ: Prentice-Hall, 1993

[8] E. Collins, S. Ghosh, and C. Scofield, "An application of a multiple neural network learning system to emulation of mortgage underwriting judgments," in Proc. IEEE Int. Conf. Neural Networks, San Diego, CA, 1998, vol. 2, pp. 459-466.

[9] S. P. Curram and J. Mingers, "Neural networks, decision tree induction and discriminant analysis: An empirical comparison," J. Oper. Res. Soc., vol. 45, pp. 440-450, 1994.

[10] G. Cybenko "Approximation by superpositions of a sigmoidal function," Math. Contr., Signals, Syst., vol. 2, pp. 303-314, 1989.

[11] A. DeMaris, Logit Modeling. Newbury Park, CA: Sage, 1992
[12] B. Dutta and S. Shekhar, "Bond rating: A nonconservative application of neural networks," in Proc. IEEE Int. Conf. Neural Networks, San Diego, CA, 1998, vol. 2, pp. 443-450.

[13] D. Garson, "Interpreting neural-network connection strengths," $A I E x$ pert, pp. 47-51, Apr. 1991.

[14] H. Guo and S. B. Gelfand, "Classification trees with neural network feature extraction," IEEE Trans. Neural Networks, vol. 3, pp. 923-933, Nov. 1992.

[15] R. F. Harrison, S. J. Marshall, and R. L. Kennedy, "The early diagnosis of heart attacks: A neuro-computational approach," in Proc. IEEE Int. Joint Conf. Neural Networks, Seattle, WA, July 1991, pt. 1, pp. 1-5.

[16] S. Haykin, Neural Networks: A Comprehensive Foundation, 1st ed. Englewood Cliffs, NJ: Prentice-Hall, 1994.

[17] J. Hertz, A. Krogh, and R. Palmer, Introduction to the Theory of Neural Computation. Reading, MA: Addison-Wesley, 1991.

[18] K. Hornik, M. Stinchcombe, and H. White, "Multilayer feedforward networks are universal approximators," Neural Networks, vol. 2, pp. 359-366, 1989.

[19] W. R. Klecka, Discriminant Analysis. Newbury Park, CA: Sage, 1980.

[20] C. Klimasauskas, "Neural networks: An engineering perspective," IEEE Commun. Mag., vol. 30, pp. 50-53, Sept. 1992.

[21] G. Lilien, "The timing of competitive market entry: An exploration study of new industrial products," Manage. Sci., vol. 36, pp. 568-585, May 1990.

[22] C. Lin and G.Lee, Neural Fuzzy Systems: A Neuro-Fuzzy Synergism to Intelligent Systems. Englewood Cliffs, NJ: Prentice-Hall, 1996.

[23] B. Mak and T. Bui, "Modeling experts consensual judgments for new product entry timing," IEEE Trans. Syst., Man, Cybern. A, vol. 26, pp. 659-667, Sept. 1996.

[24] B. Mak, T. Bui, and R. W. Blanning, "Aggregating and updating experts' knowledge: An experimental evaluation of five classification techniques," Expert Syst. Applicat., vol. 10, pp. 233-241, 1996.

[25] J. Moody and C. Darken, "Fast learning in networks of locally-tuned processing units," Neural Computat., vol. 1, pp. 281-294, 1989.

[26] R. Nath, B. Rajagopalan, and R. Ryker, "Determining the saliency of input variables in neural network classifiers," Comput. Oper. Res., vol. 24, no. 8, pp. 767-773, 1997.

[27] M. Odom and R. Sharda, "A neural network model for bankruptcy prediction," in Proc. IEEE Int. Conf. Neural Networks, San Diego, CA, 1990, pp. II163-II168.

[28] J. R. Quinlan, C4.5: Programs for Machine Learning. New York: Morgan Kaufmann, 1993.

[29] D. Rumelhart, J. McClelland, and The PDP Research Group, Parallel Distributed Processing: Explorations in The Microstructure of Cognition, Vol. I, Foundations. Cambridge, MA: MIT Press, 1986.

[30] A. P. Sage, "Systems engineering and systems management for reengineering," J. Syst. Softw., vol. 30, nos. 1-2, pp. 3-25, 1995.

[31] R. S. Shadafan and M. Niranjan, "A dynamic neural network architecture by sequential partitioning of the input space," Neural Computat., vol. 6, pp. 1202-1222, 1994.

[32] Z. Shen, M. Clarke, and R. W. Jones, "Input contribution analysis in a double input layered neural network," in Proc. Int. Conf. Artif. Neural Networks, Italy, May 1994, pp. 541-544.

[33] _ _ "A double input layered neural network—Using input weights for better understanding of decision reasoning: A medical application," in IEEE Proc. Workshop Neural Network Applicat. Tools, Liverpool, U.K., Sept. 1993, pp. 25-28.

[34] V. Subramanian, M. Hung, and M. Hu, "An experimental evaluation of neural networks for classification," Comput. Oper. Res., vol. 20, pp. 769-782, Sept. 1993

[35] K. Y. Tam and M. Kiang, "Managerial application of neural networks: The case of bank failure prediction," Manage. Sci., vol. 38, pp. 926-947, July 1992.

[36] J. Utans and J. Moody, "Selecting neural network architectures via the prediction risk: Application to corporate bond rating prediction," in IEEE Proc. 1st Int. Conf. Artif. Intell. Applicat. Wall Street, New York, Oct. 1991, pp. 35-41.

[37] R. Walrous, "Phoneme discrimination using connectionist networks," $J$. Acoust. Soc. Amer., vol. 87, pp. 1753-1772, Apr. 1990.

[38] T. Watkin, A. Rau, and M. Biehl, "The statistical mechanics of learning a rule," Amer. Phys. Soc., vol. 65, pp. 499-556, Apr. 1993.

[39] Y. Yoon, T. Guimaraes, and G. Swales, "Integrating artificial neural networks with rule-based expert systems," Decision Support Syst., vol. 11, pp. 497-507, June 1994

[40] Y. Yoon, G. Swales, and T. Margavio, "A comparison of discriminant analysis versus artificial neural networks," J. Oper. Res. Soc., vol. 44, no. 1, pp. 51-60, 1993. 